\title{
Game Coloring the Cartesian Product of Graphs
}

Xuding Zhu ${ }^{1,2}$

${ }^{1}$ DEPARTMENT OF APPLIED MATHEMATICS NATIONAL SUN YAT-SEN UNIVERSITY KAOHSIUNG, TAIWAN

E-mail: zhu@math.nsysu.edu.tw

${ }^{2}$ NATIONAL CENTER FOR THEORETICAL SCIENCES

TAIWAN

Received July 3, 2007; Revised May 19, 2008

Published online 31 July 2008 in Wiley InterScience(www.interscience.wiley.com).

DOI 10.1002/jgt.20338

Abstract: This article proves the following result: Let $G$ and $G^{\prime}$ be graphs of orders $n$ and $n^{\prime}$, respectively. Let $G^{*}$ be obtained from $G$ by adding to each vertex a set of $n^{\prime}$ degree 1 neighbors. If $G^{*}$ has game coloring number $m$ and $G^{\prime}$ has acyclic chromatic number $k$, then the Cartesian product $G \square G^{\prime}$ has game chromatic number at most $k(k+m-1)$. As a consequence, the Cartesian product of two forests has game chromatic number at most 10, and the Cartesian product of two planar graphs has game chromatic number at most 105. @ 2008 Wiley Periodicals, Inc. J Graph Theory 59: 261-278, 2008

Keywords: game chromatic number; Cartesian product; game coloring number; planar graph

\section{INTRODUCTION}

Suppose $G=(V, E)$ is a graph. The game chromatic number of $G$ is defined through a two-person game: the coloring game. Let $X$ be a set of colors. Alice and Bob

Contract grant sponsor: National Science Council; Contract grant number: NSC952115-M-110-013-MY3.

Journal of Graph Theory

(C) 2008 Wiley Periodicals, Inc. 
take turns in playing the game. Each play by either player consists of coloring an uncolored vertex of $G$ with a color from $X$. Adjacent vertices must be colored by distinct colors. The game ends if no more vertices can be colored: either because all the vertices are colored or because the uncolored vertices have no legal color, that is, for each uncolored vertex $x$, each color from $X$ is used by a neighbor of $x$. Alice wins the game if all the vertices of $G$ are successfully colored. Otherwise, Bob wins the game.

There is one more detail of the rules we need to take care of: who has the first move? This could be crucial to the outcome of the game. So depending on whether Alice or Bob has the first move, we have actually two games: The A-coloring game in which Alice has the first move, and the B-coloring game in which Bob has the first move.

We define the A-game chromatic number $\chi_{\mathrm{g}}^{A}(G)$ (respectively, the B-game chromatic number $\left.\chi_{\mathrm{g}}^{B}(G)\right)$ of $G$ to be the least number of colors in a color set $X$ for which Alice has a winning strategy in the A-coloring game on $G$ (respectively, the B-coloring game on $G)$. There are graphs $G$ for which $\chi_{\mathrm{g}}^{A}(G)$ and $\chi_{\mathrm{g}}^{B}(G)$ are far apart. Let $G=K_{n, n}-I$, where $I$ is a one-factor of the complete bipartite graph $K_{n, n}$. Let $H$ be obtained from $G$ by adding an isolated vertex. It is easy to verify that $\chi_{\mathrm{g}}^{B}(H)=\chi_{\mathrm{g}}^{A}(G)=n$ and $\chi_{\mathrm{g}}^{A}(H)=\chi_{\mathrm{g}}^{B}(G)=2$.

The coloring game on planar maps was invented by Brams, and was published by Gardner [12] in his column "Mathematical Games" in Scientific American in 1981. It remained unnoticed by the graph-theoretic community until 10 years later, when it was reinvented by Bodlaender [3]. Bodlaender defined the game chromatic number of graphs, and conjectured that the game chromatic numbers of planar graphs are bounded by a constant. Since then the problem has attracted considerable attention and the game chromatic numbers of various classes of graphs have been studied (see [2] for a recent survey).

In the literature on game chromatic number, the game is usually defined in such a way that Alice has the first move, except that in [5], the game list coloring in which Bob has the first move was also considered. Thus the study of game coloring of graphs is concentrated in the A-game chromatic number of graphs, which is simply called the game chromatic number, and denoted by $\chi_{\mathrm{g}}(G)$ in the literature. The fact that the game is defined with Alice having the first move is incidental. It is for the purpose of convenience that we always let Alice have the first move. However, for the proofs in this article, we need to use results about coloring games (and marking games, which are defined in the next section) in which Bob has the first move.

Suppose $\mathcal{H}$ is a class of graphs. Let

$$
\chi_{\mathrm{g}}^{A}(\mathcal{H})=\max \left\{\chi_{\mathrm{g}}^{A}(G): G \in \mathcal{H}\right\}
$$

and

$$
\chi_{\mathrm{g}}^{B}(\mathcal{H})=\max \left\{\chi_{\mathrm{g}}^{B}(G): G \in \mathcal{H}\right\} .
$$


Although for a single graph $G$, there can be a big difference between $\chi_{\mathrm{g}}^{A}(G)$ and $\chi_{\mathrm{g}}^{B}(G)$, we shall show that for natural classes of graphs, $\chi_{\mathrm{g}}^{A}(\mathcal{H})=\chi_{\mathrm{g}}^{B}(\mathcal{H})$. For a graph $G$, let $2 G$ denote the union of two disjoint copies of $G$, and $G^{+}$denote the graph obtained from $G$ by adding an isolated vertex.

Lemma 1. Suppose $\mathcal{H}$ is a class of graphs such that if $G \in \mathcal{H}$, then $2 G \in \mathcal{H}$ and $G^{+} \in \mathcal{H}$. Then $\chi_{\mathrm{g}}^{A}(\mathcal{H})=\chi_{\mathrm{g}}^{B}(\mathcal{H})$.

Proof. Assume $\chi_{\mathrm{g}}^{B}(\mathcal{H})=k$. Then there is a graph $G \in \mathcal{H}$ such that $\chi_{\mathrm{g}}^{B}(G)>$ $k-1$, that is, with a set $X$ of $k-1$ colors, Bob has a strategy, called BWIN-Strategy, to win the B-coloring game on $G$ with color set $X$.

Assume first that $G$ has an odd number of vertices. We shall show that Bob has a winning strategy for the A-coloring game on $2 G$ with color set $X$. Let the two copies of $G$ in $2 G$ be $G_{1}$ and $G_{2}$. Assume Alice colors a vertex of $G_{1}$ in her first move. Then Bob colors a vertex of $G_{2}$ in his first move, according to BWIN-Strategy. From then on, whenever Alice colors a vertex of $G_{2}$, Bob also colors a vertex of $G_{2}$, using BWIN-Strategy. If Alice colors a vertex of $G_{1}$, then Bob colors an arbitrary vertex of $G_{1}$ with any legal color. Because $G_{1}$ has an odd number of vertices, whenever Alice colors a vertex of $G_{1}$, Bob can find an uncolored vertex in $G_{1}$ and colors it. Thus Bob wins the game. If $G$ has an even number of vertices, the same argument as above shows that Bob has a winning strategy for the A-coloring game on $(2 G)^{+}$with color set $X$. This proves that $\chi_{\mathrm{g}}^{A}(\mathcal{H}) \geq k$

Assume $\chi_{\mathrm{g}}^{A}(\mathcal{H})=k$. Then there is a graph $G \in \mathcal{H}$ such that $\chi_{\mathrm{g}}^{A}(G)>k-$ 1 , that is, with a set $X$ of $k-1$ colors, Bob has a strategy to win the Acoloring game on $G$ with color set $X$. Then Bob has a strategy to win the B-coloring game on $G^{+}$, because Bob can simply color the added isolated vertex in his first move, and then use his winning strategy for the A-coloring game afterward. This proves that $\chi_{\mathrm{g}}^{B}(\mathcal{H}) \geq k$, and hence completes the proof of Lemma 1.

For classes $\mathcal{H}$ of graphs satisfying the assumption of Lemma 1, we shall denote the common value of $\chi_{\mathrm{g}}^{A}(\mathcal{H})$ and $\chi_{\mathrm{g}}^{B}(\mathcal{H})$ by $\chi_{\mathrm{g}}(\mathcal{H})$.

We denote by $\mathcal{F}$ the family of forests, by $\mathcal{I}_{k}$ the family of interval graphs with clique number $k$, by $\mathcal{P}$ the family of planar graphs, by $\mathcal{Q}$ the family of outer planar graphs, by $\mathcal{P} T_{k}$ the family of partial $k$-trees. It is proved by Faigle et al. [11] that $\chi_{\mathrm{g}}(\mathcal{F})=4$, proved by Faigle et al. [11] that $\chi_{\mathrm{g}}\left(\mathcal{I}_{k}\right) \leq 3 k-2$, proved by Guan and Zhu [14] and Kierstead and Trotter [19] that $6 \leq \chi_{\mathrm{g}}(\mathcal{Q}) \leq 7$, and proved by Zhu [28] that $\chi_{\mathrm{g}}\left(\mathcal{P} T_{k}\right) \leq$ $3 k+2$ for $k \geq 2$, proved by Zhu [29] and Kierstead and Trotter [19] that $8 \leq \chi_{\mathrm{g}}(\mathcal{P}) \leq 17$

In this article, we are interested in the game chromatic number of the Cartesian product of graphs. Given two graphs $G=(V, E)$ and $G^{\prime}=\left(V^{\prime}, E^{\prime}\right)$, the Cartesian product $G \square G^{\prime}$ of $G$ and $G^{\prime}$ has vertex set $V \times V^{\prime}=\left\{\left(x, x^{\prime}\right): x \in V, x^{\prime} \in V^{\prime}\right\}$, and in which $\left(x, x^{\prime}\right)$ is adjacent to $\left(y, y^{\prime}\right)$ if either $x=y$ and $x^{\prime} y^{\prime} \in E^{\prime}$ or $x y \in E$ and 
$x^{\prime}=y^{\prime}$. Many coloring parameters of graph products have been studied extensively in the literature. It is known and easy to see that for any graphs $G, G^{\prime}, \chi\left(G \square G^{\prime}\right)=$ $\max \left\{\chi(G), \chi\left(G^{\prime}\right)\right\}$. One natural question is how the game chromatic number of $G \square G^{\prime}$ relates to various parameters of $G$ and $G^{\prime}$.

The game chromatic number of the Cartesian product of graphs was first studied in [1]. The exact values of $\chi_{\mathrm{g}}^{A}\left(K_{2} \square P_{n}\right)$ and $\chi_{\mathrm{g}}^{A}\left(K_{2} \square K_{n}\right)$ are determined. It is also proved in [1] that the Cartesian product of two forests has game chromatic number at most 12 and the Cartesian product of two planar graphs has game chromatic number at most 650 .

In this article, we show that $\chi_{\mathrm{g}}^{B}\left(G \square G^{\prime}\right)$ is bounded from above by a function of the game coloring number of $G^{*}$ (a variation of $\chi_{\mathrm{g}}^{B}\left(G^{*}\right)$ that will be defined in the next section) and the acyclic chromatic number of $G^{\prime}$ (a variation of the chromatic number which will be defined in Section 3). Here $G^{*}$ is obtained from $G$ by adding some degree 1 vertices, which usually do not change the game coloring number, that is, usually $G$ and $G^{*}$ have the same game coloring number. As a consequence of this result, we obtain upper bounds for the game chromatic number of the Cartesian product of various classes of graphs. For example, the Cartesian product of two forests has game chromatic number at most 10 , the game chromatic number of the Cartesian product of two planar graphs is at most 105, the game chromatic number of the Cartesian product of two outerplanar graphs is at most 27 , the game chromatic number of the Cartesian product of a planar graph and an outerplanar graph is at most 55.

\section{GAME COLORING NUMBER AND ITS VARIATIONS}

To obtain upper bounds for the game chromatic number of the Cartesian product of graphs, we need to use the game coloring number of graphs and its variations.

Suppose $G=(V, E)$ is a graph. The game coloring number of $G$ is also defined through a two-person game: the marking game. Alice and Bob take turns in playing the game. Each play by either player consists of marking an unmarked vertex of $G$. The game ends when all vertices are marked. For a vertex $x$ of $G$, let $b(x)$ be the number of neighbors of $x$ that are marked before $x$ is marked. The score of the game is

$$
s=1+\max _{x \in V(G)} b(x)
$$

Alice's goal is to minimize the score, while Bob's goal is to maximize it.

Again, depending on whether Alice or Bob has the first move, we have two marking games. The game coloring number (Bob-first) $\operatorname{col}_{\mathrm{g}}^{B}(G)$ (respectively, the game coloring number (Alice-first) $\operatorname{col}_{\mathrm{g}}^{A}(G)$ ) of $G$ is the least $s$ such that Alice has a strategy that results in a score at most $s$ in the marking game on $G$ in which Bob has the first move (respectively, Alice has the first move). 
The game coloring number of a graph was first formally introduced in [27] as a tool in the study of the game chromatic number of graphs. However, it is of independent interest. The concept itself and some of its variations have been studied extensively in the literature $[2,8,9,14,15,17,20-24,26,28,29]$.

Again in the definition of the marking game in other articles, Alice is assumed to have the first move. So the study on game coloring number concentrated on the parameter $\operatorname{col}_{\mathrm{g}}^{A}(G)$. However, it is not difficult to show that for any graph $G$, $\operatorname{col}_{g}^{A}(G)$ and $\operatorname{col}_{g}^{B}(G)$ differ by at most one. Moreover, when we study upper bounds on the game coloring number for natural classes of graphs, whether Alice or Bob has the first move does not really matter. Suppose $\mathcal{H}$ is a class of graphs. Let

$$
\operatorname{col}_{\mathrm{g}}^{A}(\mathcal{H})=\max \left\{\operatorname{col}_{\mathrm{g}}^{A}(G): G \in \mathcal{H}\right\},
$$

and

$$
\operatorname{col}_{\mathrm{g}}^{B}(\mathcal{H})=\max \left\{\operatorname{col}_{\mathrm{g}}^{B}(G): G \in \mathcal{H}\right\}
$$

Lemma 2. Suppose $\mathcal{H}$ is a class of graphs such that if $G \in \mathcal{H}$, then $2 G \in \mathcal{H}$ and $G^{+} \in \mathcal{H}$. Then $\operatorname{col}_{\mathrm{g}}^{A}(\mathcal{H})=\operatorname{col}_{\mathrm{g}}^{B}(\mathcal{H})$.

The proof of Lemma 2 is parallel to that of Lemma 1 and is omitted. For classes $\mathcal{H}$ of graphs satisfying the assumption of Lemma 2, we shall denote the common value of $\operatorname{col}_{\mathrm{g}}^{A}(\mathcal{H})$ and $\operatorname{col}_{\mathrm{g}}^{B}(\mathcal{H})$ by $\operatorname{col}_{\mathrm{g}}(\mathcal{H})$.

The exact value of the game coloring numbers of $\mathcal{F}, \mathcal{I}_{k}, \mathcal{Q}$ and $\mathcal{P} T_{k}$ are known. It is proved by Faigle et al. [11] that $\operatorname{col}_{\mathrm{g}}(\mathcal{F})=4$, proved by Faigle et al. [11] and Kierstead and Yang [23] that $\operatorname{col}_{\mathrm{g}}\left(\mathcal{I}_{k}\right)=3 k-2$, proved by Guan and Zhu [14] and Kierstead and Yang [23] that $\operatorname{col}_{\mathrm{g}}(\mathcal{Q})=7$, and proved by Zhu [28] and $\mathrm{Wu}$ and Zhu [26] that $\operatorname{col}_{\mathrm{g}}\left(\mathcal{P} T_{k}\right)=3 k+2$ for $k \geq 2$. It is also known $[29,26]$ that $11 \leq \operatorname{col}_{\mathrm{g}}(\mathcal{P}) \leq 17$.

It is easy to see that for any graph $G, \chi_{\mathrm{g}}^{B}(G) \leq \operatorname{col}_{\mathrm{g}}^{B}(G)$ and $\chi_{\mathrm{g}}^{A}(G) \leq \operatorname{col}_{\mathrm{g}}^{A}(G)$. For many natural classes of graphs, the best known upper bounds for their game chromatic numbers are obtained by finding upper bounds for their game coloring numbers.

In the study of the game chromatic number of the Cartesian product of graphs, we need a variation of the game coloring number of graphs. The parameter we need is also defined through a marking game on $G$, which is similar to the marking game defined above, except that Bob will have some small privilege when he makes a move. Any change of the rules could have significant impact on the game. We shall modify the rules slowly, by inserting some intermediate games. These intermediate games may be of independent interest, however, our main purpose of introducing these games is to make the proofs more transparent.

P-Marking game. The $P$-marking game on $G$ is the same as the Bob-first marking game on $G$, with one small change in the rules: Bob is allowed to pass a move, that 
is, in Bob's turn, he is allowed to mark no vertex at all. Alice does not have this privilege. She must mark exactly one vertex on each of her move. The score of the game is defined in the same way as in the marking game. The P-game coloring number $\operatorname{Pcol}_{\mathrm{g}}(G)$ of $G$ is the smallest integer $s$ such that Alice has a strategy that results in a score at most $s$ in the P-marking game on $G$.

It follows from the definition that for any $\operatorname{graph} G, \operatorname{Pcol}_{\mathrm{g}}(G) \geq \operatorname{col}_{\mathrm{g}}^{B}(G)$, because the P-marking game only gives some privilege to Bob. Hence he can do at least as well as in the marking game. We shall show that Bob cannot really take advantage from the change of rules. To be precise, we have the following result.

Lemma 3. For any graph $G, \operatorname{Pcol}_{\mathrm{g}}(G)=\operatorname{col}_{\mathrm{g}}^{B}(G)$.

Proof. It suffices to show that $\operatorname{Pcol}_{\mathrm{g}}(G) \leq \operatorname{col}_{\mathrm{g}}^{B}(G)$. Assume Alice has a strategy for playing the marking game on $G$ that ensures the score of the game is at most $s$. Assume now Alice and Bob play the P-marking game on $G$. We shall show that Alice has a strategy to ensure that the score of the game is at most $s$. Alice will pretend that she is playing the marking game on $G$. So there will be two games played at the same time: the real game, and the pretend game. The vertex Alice chooses to mark in the pretend game will determine the vertex she marks in the real game. We say a vertex is $R$-marked if it is marked in the real game, and a vertex is $P$-marked if it is marked in the pretend game. It will be the case that every R-marked vertex is a P-marked vertex. However, a P-marked vertex may be not R-marked.

Let $Q$ be a "dynamic" set of vertices constructed as follows: At the beginning of the game, $Q=\varnothing$. If a vertex $v$ is P-marked but not R-Marked, then add $v$ to $Q$. So in the process of the game, the number of vertices in $Q$ never decreases.

If Bob does not mark any vertex in a move, or he marks a vertex in $Q$ in a move, then we say Bob played a passing move. Suppose Bob has made a move. If the move is not a passing move, then Alice responds to that move by using her strategy for the pretend marking game. Assume Bob made a passing move. That is, either Bob did not mark any vertex in the move, or he marked a vertex which is P-marked. Thus Alice cannot respond to that move by using her strategy for the pretend marking game, because the strategy only tells Alice what to do if Bob marks a not yet Pmarked vertex. Let $U$ be the set of unmarked vertices in the pretend game. If $U=\emptyset$, then Alice plays arbitrarily till the end of the real P-marking game. Assume $U \neq \emptyset$. Let $x \in U$ be a vertex which has minimum degree in $G$ among all vertices of $U$. Alice pretends that Bob has just marked $x$ (hence add $x$ to $Q$ ), and responds to the move according to her strategy in the pretend marking game.

After both games (the real game and the pretend game) are completed, we obtain two orderings of the vertices of $G,<_{R}$ and $<_{P}: x<_{R} y$ if $x$ is marked before $y$ in the real game, and $x<_{P} y$ if $x$ is marked before $y$ in the pretend game. We write $u \sim v$ if $u$ and $v$ are adjacent. By our assumption, for any vertex $u$,

$$
\left|\left\{v: v \sim u, v<_{P} u\right\}\right| \leq s-1 .
$$


We need to show that for any vertex $u$,

$$
\left|\left\{v: v \sim u, v<_{R} u\right\}\right| \leq s-1
$$

Let $w$ be the largest vertex in $<_{P}$, that is, the last marked vertex in the pretend game. If $u \notin Q$, then we have

$$
\left\{v: v \sim u, v<_{R} u\right\} \subseteq\left\{v: v \sim u, v<_{P} u\right\}
$$

because at any moment, any marked vertex in the real game is a marked vertex in the pretend game. Therefore, $\left|\left\{v: v \sim u, v<_{R} u\right\}\right| \leq s-1$.

If $u \in Q$, then at the moment $u$ is added to $Q, u$ has the minimum degree among all unmarked vertices. In particular, $d_{G}(u) \leq d_{G}(w)$. As $w$ is the last marked vertex in the pretend game, we have $\left|\left\{v: v \sim w, v<_{P} w\right\}\right|=d_{G}(w) \leq s-1$. Hence $\mid\{v$ : $\left.v \sim u, v<_{R} u\right\} \mid \leq d_{G}(u) \leq s-1$. This completes the proof of Lemma 3 .

B-Marking game. The $B$-marking game on $G$ is the same as the P-marking game on $G$, with one more privilege to Bob. In his turn, he can mark an arbitrary number of vertices with a red pen, and marks at most one vertex with a black pen. Alice marks exactly one vertex with a black pen on each of her move. For a vertex $x$ of $G$, let $b(x)$ be the number of black neighbors of $x$ that are marked before $x$ is marked. The score of the game is

$$
s=1+\max _{x \in V(G)} b(x)
$$

The B-game coloring number $\operatorname{Bcol}_{\mathrm{g}}(G)$ of $G$ is the least $s$ such that Alice has a strategy that results in a score at most $s$. So only black neighbors are counted in the calculation of $b(x)$. The purpose of Bob marking a vertex by red pen is to forbidden this vertex to be marked in black.

In the B-marking game, Bob can do at least as well as in the P-marking game. So for any graph $G, \operatorname{Bcol}_{\mathrm{g}}(G) \geq \operatorname{Pcol}_{\mathrm{g}}(G)$. We shall show that Bob cannot take advantage from the extra privilege.

Lemma 4. For any graph $G, \operatorname{Bcol}_{\mathrm{g}}(G)=\operatorname{Pcol}_{\mathrm{g}}(G)$.

Proof. Assume Alice has a strategy for the P-marking game that ensures the score of the game is at most $s$. Assume now that Alice and Bob play the B-marking game. Alice will pretend that she is playing the P-marking game, and ignore all vertices marked in red. That is, if a vertex is marked in red by Bob, Alice pretends that the vertex is unmarked yet. If, by following her strategy, Alice needs to mark a vertex $x$, but $x$ is marked in red already (this can happen because in Alice's pretend game, the vertex $x$ is unmarked yet), then she pretends that she marked $x$ in her current turn, and then pretends that Bob passed his next turn. So it is Alice's turn again in the pretend game. 
After both the real game and the pretend game are completed, let $x<_{R} y$ if $x$ is marked before $y$ in the real game, and $x<_{P} y$ if $x$ is marked before $y$ in the pretend game. It is easy to verify that for any vertex $u$,

$$
\left\{v: v \sim u, v<_{R} u, v \text { is marked black }\right\} \subseteq\left\{v: v \sim u, v<_{P} u\right\} .
$$

Therefore, $\mid\left\{v: v \sim u, v<_{R} u, v\right.$ is marked black $\} \mid \leq s-1$.

R-Marking game. The $R$-marking game on $G$ is the same as the $\mathrm{B}$-marking game on $G$, with still one more privilege to Bob. Before the game starts, Bob is allowed to reserve a set $Z$ of vertices. Alice is not allowed to mark any vertex in $Z$. Only Bob can mark vertices in $Z$. The game ends when all vertices of $V(G) \backslash Z$ are marked. For a vertex $x \in V(G) \backslash Z$, let $b(x)$ be the number of black neighbors of $x$ that are marked before $x$ is marked. The score of the game is

$$
s=1+\max _{x \in V(G) \backslash Z} b(x) .
$$

The $R$-game coloring number $\operatorname{Rcol}_{\mathrm{g}}(G)$ of $G$ is the least $s$ such that Alice has a strategy that results in a score at most $s$.

As Bob may reserve no vertices at the beginning of the game, in the R-marking game, he can do at least as well as in the B-marking game. So for any graph $G$, $\operatorname{Rcol}_{\mathrm{g}}(G) \geq \mathrm{Bcol}_{\mathrm{g}}(G)$. We shall show that Bob cannot take advantage from the extra privilege.

Lemma 5. For any graph $G, \operatorname{Rcol}_{\mathrm{g}}(G)=\operatorname{Bcol}_{\mathrm{g}}(G)$.

Proof. Assume Alice has a strategy for playing the $B$-marking on $G$ that ensures the score of the game is at most $s$. Assume now Alice and Bob play the R-marking game on $G$. Alice will pretend that she is playing the B-marking game on $G$. If Alice, by following her strategy for the B-marking game, needs to mark a vertex $x \in Z$. Then Alice pretends that she marked the vertex $x$, and pretends that in Bob's next turn, he passed without marking any vertex, and so it is Alice's turn again. Note that in the real game, the vertex $x$ is still unmarked, but in the pretend game, it is marked already. If in a later move, Bob marks a vertex $x \in Z$ which in the pretend game has already been marked earlier by Alice, then Alice pretends that Bob passed the move without marking any vertex. Similarly, let $x<_{R} y$ if $x$ is marked before $y$ in the real game, and $x<_{P} y$ if $x$ is marked before $y$ in the pretend game. It is easy to verify that for any vertex $u \in V(G) \backslash Z$,

$$
\left\{v: v \sim u, v<_{R} u, v \text { is marked black }\right\} \subseteq\left\{v: v \sim u, v<_{P} u\right\}
$$

Hence the score of the real R-marking game is at most $s$.

Corollary 1. For any graph $G, \operatorname{col}_{\mathrm{g}}^{B}(G)=\operatorname{Rcol}_{\mathrm{g}}(G)$. 


\section{CARTESIAN PRODUCT OF GRAPHS}

In this section, we prove the main result of the article by presenting a bound for $\chi_{\mathrm{g}}^{B}\left(G \square G^{\prime}\right)$ in terms of $\operatorname{col}_{\mathrm{g}}^{B}\left(G^{*}\right)$ and $\chi_{a}\left(G^{\prime}\right)$, where $G^{*}$ is obtained from $G$ by adding some degree 1 vertices. The parameter $\chi_{a}\left(G^{\prime}\right)$ is the acyclic chromatic number of $G^{\prime}$ defined as follows: An acyclic k-coloring of a graph $G$ is a mapping $f: V(G) \rightarrow\{1,2, \ldots, k\}$ such that for any $j, f^{-1}(j)$ is an independent set, and for any $j, j^{\prime}$, the subgraph of $G$ induced by $f^{-1}(j) \cup f^{-1}\left(j^{\prime}\right)$ is a forest. The acyclic chromatic number $\chi_{a}(G)$ of $G$ is the minimum $k$ for which $G$ has an acyclic $k$ coloring. The acyclic chromatic number of graphs was first introduced by Grünbaum [13] and has been studied extensively in the literature. In particular, it is proved by Borodin [4] that planar graphs have acyclic chromatic number at most 5. It is proved by DeVos et al. [6] that for any proper minor closed class $\mathcal{H}$ of graphs, there is a constant $\kappa_{H}$ such that every graph $G \in \mathcal{H}$ has acyclic chromatic number at most $\kappa_{H}$.

For our purpose, we actually need a parameter bounded above by $\chi_{a}(G)$ : the weak-acyclic chromatic number, which was defined by Kierstead [18]. A weak acyclic k-coloring of a graph $G$ is a mapping $f: V(G) \rightarrow\{1,2, \ldots, k\}$ such that for any $j, f^{-1}(j)$ is an independent set, and for any $j, j^{\prime}$, the subgraph of $G$ induced by $f^{-1}(j) \cup f^{-1}\left(j^{\prime}\right)$ has an orientation in which each vertex has outdegree at most 1 . In other words, each component of the subgraph of $G$ induced by $f^{-1}(j) \cup f^{-1}\left(j^{\prime}\right)$ is either a tree or contains one cycle. The weak acyclic chromatic number $\chi_{w a}(G)$ of $G$ is the minimum $k$ for which $G$ has a weak acyclic $k$-coloring.

Theorem 1. Let $G$ and $G^{\prime}$ be two graphs. Assume $G^{\prime}$ has $n^{\prime}$ vertices. Let $G^{*}$ be obtained from $G$ by adding to each vertex $x$ of $G$ a set $W_{x}$ of $n^{\prime}$ degree 1 neighbors. That is, $\left|W_{x}\right|=n^{\prime}$ and each vertex $w \in W_{x}$ is adjacent to $x$ and adjacent to no other vertex. Suppose $\operatorname{Rcol}\left(G^{*}\right)=m$ and $\chi_{w a}\left(G^{\prime}\right)=k$. Then $\chi_{\mathrm{g}}^{B}\left(G \square G^{\prime}\right) \leq k(k+m-1)$.

Proof. Assume $V(G)=\left\{x_{1}, x_{2}, \ldots, x_{n}\right\}$ and $V\left(G^{\prime}\right)=\left\{y_{1}, y_{2}, \ldots, y_{n^{\prime}}\right\}$. The vertices of $G \square G^{\prime}$ are $\left\{\left(x_{i}, y_{j}\right): 1 \leq i \leq n, 1 \leq j \leq n^{\prime}\right\}$.

For $i=1,2, \ldots, n$, let $V_{i}=\left\{\left(x_{i}, y_{j}\right): 1 \leq j \leq n^{\prime}\right\}$ and for $j=1,2, \ldots, n^{\prime}$, let $U_{j}=\left\{\left(x_{i}, y_{j}\right): 1 \leq i \leq n\right\}$. We also use $V_{i}$ (respectively, $\left.U_{j}\right)$ to denote the subgraph of $G \square G^{\prime}$ induced by $V_{i}$ (respectively, $U_{j}$ ). Thus each $V_{i}$ is a copy of $G^{\prime}$ and each $U_{j}$ is a copy of $G$.

Usually we view the product graph $G \square G^{\prime}$ as drawn in the shape of a rectangle. Each $V_{i}$ is drawn in a horizontal line, and each $U_{j}$ is drawn in a vertical line. So each horizontal line is a copy of $G^{\prime}$, and each vertical line is a copy of $G$.

Let $\phi: V\left(G^{\prime}\right) \rightarrow\{1,2, \ldots, k\}$ be a weak acyclic $k$-coloring of $G^{\prime}$. Each $V_{i}$ is a copy of $G^{\prime}$. The coloring $\phi$ induces a weak acyclic $k$-coloring of each $V_{i}$, also denoted by $\phi$. For any two colors $a, b \in\{1,2, \ldots, k\}$, for any $i=1,2, \ldots, n$, let $V_{i}^{a, b}$ be the subgraph of $V_{i}$ induced by the union of the two color classes $\phi^{-1}(a) \cup$ $\phi^{-1}(b)$. As $\phi$ is a weak acyclic coloring, $V_{i}^{a, b}$ has an orientation in which each vertex has at most one out-neighbor. This defines an orientation of "horizontal edges" of Journal of Graph Theory DOI 10.1002/jgt 
$G \square G^{\prime}$. Each vertex has at most one out-neighbor in each of the other classes. So each vertex has out-degree at most $k-1$.

For the coloring game on $G \square G^{\prime}$, we use colors $(a, b): a=1,2, \ldots, k, b=$ $1,2, \ldots, k+m-1$. For $a=1,2, \ldots, k$, let $C_{a}=\{(a, b): b=1,2, \ldots, k+$ $m-1\}$.

For each vertex $\left(x_{i}, y_{j}\right)$ of $G \square G^{\prime}$, if $\phi\left(y_{j}\right)=a$ then the color set $C_{a}$ is called the set of correct colors for $\left(x_{i}, y_{j}\right)$. When Alice colors a vertex $\left(x_{i}, y_{j}\right)$, she always uses a correct color. Of course, Bob may use any color. In the following, we shall just give a strategy for Alice to find a vertex to be colored. Once the vertex is selected, she will color it with any correct color.

By our assumption, Alice has a strategy to play the R-marking game on $G^{*}$ so that the score is at most $m$. We call this strategy A-strategy.

There are $n^{\prime}$ copies of $G$ in the product $G \square G^{\prime}$ induced by the $U_{j}$ 's (for $j=$ $\left.1,2, \ldots, n^{\prime}\right)$. From these $U_{j}$ 's, we construct $n^{\prime}$ copies of $G^{*}$. The copy made from $U_{j}$ is denoted by $U_{j}^{*}$. The vertex set of $G^{*}$ is $V(G) \cup\left(\cup_{i=1}^{n} W_{x_{i}}\right)$. So

$$
U_{j}^{*}=U_{j} \cup\left(\cup_{i=1}^{n}\left(W_{x_{i}} \times\left\{y_{j}\right\}\right)\right)
$$

There will be $n^{\prime}$ R-marking games played at the same time. Alice[ $\left.j\right]$ and $\operatorname{Bob}[j]$ play the R-marking game on $U_{j}^{*}$.

Let $Z=V\left(G^{*}\right) \backslash V(G)$. For each $j, \operatorname{Bob}[j]$ reserves $Z \times\left\{y_{j}\right\}$ before the start of the game. The moves on all these R-marking games are determined by the single coloring game played on $G \square G^{\prime}$, and vice versa. Alice's strategy in the coloring game on $G \square G^{\prime}$ is determined as in the following flow chart:

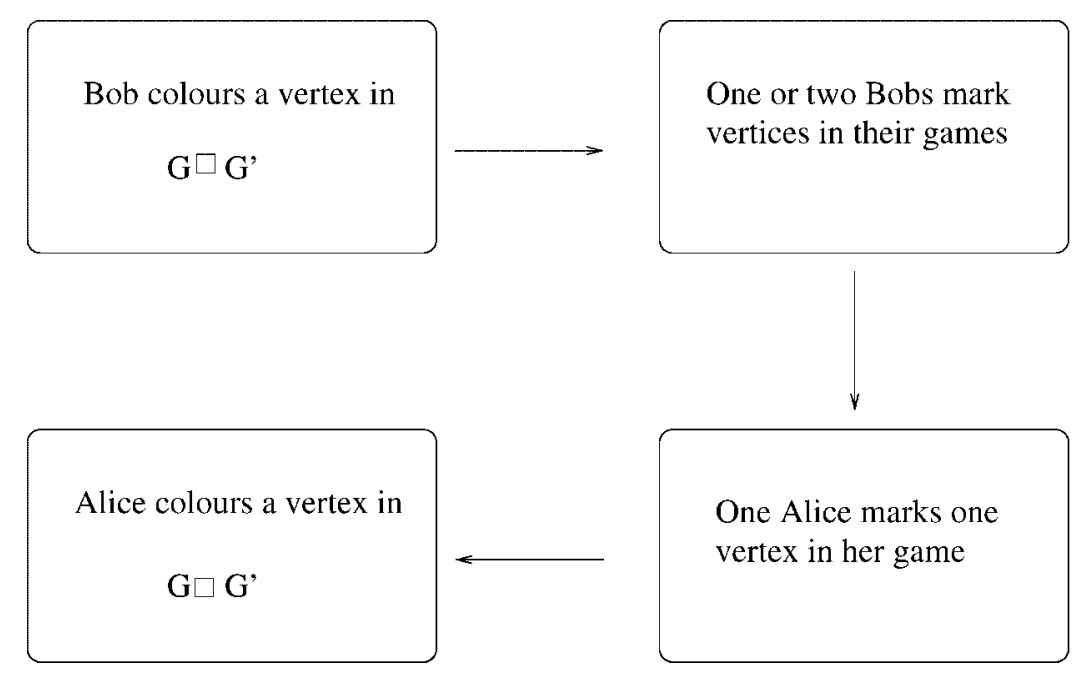

FIGURE 1. The flow chart for Alice to decide her next move in the coloring game. 
Suppose Bob colored a vertex in $G \square G^{\prime}$. This provokes one or two of the Bobs (in the group of marking games) to mark vertices. However, at most one of the Bobs will mark a vertex in black. One of the Alices (in the group marking games) respond to the marking in black (if there is one), according to A-strategy. That determines Alice's move in the coloring game on $G \square G^{\prime}$.

Here are the details: Suppose Bob has just colored a vertex $\left(x_{i}, y_{j}\right)$ with a color $(a, q)$. If $\phi\left(y_{j}\right)=a$, that is, Bob used a correct color for the vertex $\left(x_{i}, y_{j}\right)$, then $\operatorname{Bob}[j]$ marks the vertex $\left(x_{i}, y_{j}\right) \in U_{j}^{*}$ in black. In the R-marking game played on $U_{j}^{*}$, it is Alice's turn. Alice[ $\left.j\right]$ uses A-strategy to find a vertex $\left(x_{i^{\prime}}, y_{j}\right) \in U_{j}^{*}$ to be marked. The corresponding move in the coloring game of $G \square G^{\prime}$ is that Alice will color $\left(x_{i^{\prime}}, y_{j}\right)$ in her current move.

Assume $\phi\left(y_{j}\right)=b$ and $a \neq b$, that is, Bob used an incorrect color for vertex $\left(x_{i}, y_{j}\right)$. Then consider the oriented subgraph $V_{i}^{a, b}$. The vertex $\left(x_{i}, y_{j}\right)$ has at most one out-neighbor. If $\left(x_{i}, y_{j}\right)$ has no out-neighbor in $V_{i}^{a, b}$, then $\operatorname{Bob}[j]$ marks the vertex $\left(x_{i}, y_{j}\right) \in U_{j}^{*}$ in red, and Bob $[j]$ decides not to mark any vertex in black at this turn. So in the R-marking game played on $U_{j}^{*}$, it is Alice's turn. Alice $[j]$ uses A-strategy to find a vertex $\left(x_{i^{\prime}}, y_{j}\right) \in U_{j}^{*}$ to be marked. The corresponding move in the coloring game of $G \square G^{\prime}$ is that Alice will color $\left(x_{i^{\prime}}, y_{j}\right)$ in her current move.

Assume $\left(x_{i}, y_{j}\right)$ has one out-neighbor $\left(x_{i}, y_{j^{\prime}}\right) \in V_{i}^{a, b}$. Then Bob[j] marks the vertex $\left(x_{i}, y_{j}\right) \in U_{j}^{*}$ in red, but Bob $[j]$ has not completed his current move yet (recall that Bob can mark an arbitrary number of vertices in red and at most one vertex in black in his turn). So in the R-marking game played on $U_{j}^{*}$, it is not yet Alice's turn.

At the same time, in the R-marking game played on $U_{j^{\prime}}^{*}$, Bob $\left[j^{\prime}\right]$ marks one vertex $\left(w, y_{j^{\prime}}\right) \in W_{x_{i}} \times\left\{y_{j^{\prime}}\right\}$ in black. So in the R-marking game played on $U_{j^{\prime}}^{*}$, it is Alice's turn. Alice[ $\left.j^{\prime}\right]$ uses A-strategy to find a vertex $\left(x_{i^{\prime}}, y_{j^{\prime}}\right)$ to be marked in her current move. The corresponding move in the coloring game of $G \square G^{\prime}$ is that Alice will color $\left(x_{i^{\prime}}, y_{j^{\prime}}\right)$ in her current move.

The above description indeed gives a strategy for Alice to find a vertex to color in the coloring game played on $G \square G^{\prime}$.

Now we shall show that by using the above strategy, at any moment of the game, for any uncolored vertex $\left(x_{s}, y_{t}\right)$ of $G \square G^{\prime}$, including the vertex chosen by Alice to color in her current move, there is a correct color for $\left(x_{s}, y_{t}\right)$.

Fix a moment of the game. Assume $\left(x_{s}, y_{t}\right)$ is not colored yet at the moment. Assume $\phi\left(y_{t}\right)=a$ (and hence $C_{a}$ is the set of correct colors for $\left(x_{s}, y_{t}\right)$ ). We shall show that there is a color in $C_{a}$ that is not used by any neighbor of $\left(x_{s}, y_{t}\right)$.

Let $K=\left\{\left(x_{i}, y_{j}\right):\left(x_{i}, y_{j}\right) \sim\left(x_{s}, y_{t}\right),\left(x_{i}, y_{j}\right)\right.$ is colored with a color from $\left.C_{a}\right\}$.

We shall prove $|K| \leq k+m-2$. Since $\left|C_{a}\right|=k+m-1$, it would follow that there is a color in $C_{a}$ that is not used by any neighbor of $\left(x_{s}, y_{t}\right)$.

The set $K$ of colored neighbors of $\left(x_{s}, y_{t}\right)$ is divided into two groups: the horizontal neighbors of the form $\left(x_{s}, y_{j}\right) \in V_{s}$ and the vertical neighbors of the form $\left(x_{i}, y_{t}\right) \in$ $U_{t}$. By our strategy, each vertical neighbor of $\left(x_{s}, y_{t}\right) \in K$ is a vertex in $U_{t}^{*}$ marked in black (observe that only incorrectly colored vertices are ever marked red). The horizontal neighbors of $\left(x_{s}, y_{t}\right)$ in $K$ are further divided into two smaller groups: the 
in-neighbors of $\left(x_{s}, y_{t}\right)$ and the out-neighbors of $\left(x_{s}, y_{t}\right)$ (recall that the horizontal edges are oriented). By our assumption, $\left(x_{s}, y_{t}\right)$ has at most one out-neighbor in $V_{s}^{a, b}$ for each $b \neq a$. So $\left(x_{s}, y_{t}\right)$ has at most $k-1$ out-neighbors. By our strategy, each in-neighbor of $\left(x_{s}, y_{t}\right) \in K$ corresponds to one vertex in $W_{x_{s}} \times\left\{y_{t}\right\}_{\subseteq} U_{t}^{*}$ marked in black. So the total number of vertices in $K$ is at most $k-1$ plus the number of black neighbors of $\left(x_{s}, y_{t}\right)$ in $U_{t}^{*}$. As Alice is using A-strategy for the R-marking game on $U_{t}^{*},\left(x_{s}, y_{t}\right)$ has at most $m-1$ black neighbors in $U_{t}^{*}$. Therefore $|K| \leq$ $(m-1)+(k-1)=m+k-2$. This completes the proof of Theorem 1.

It is well known (as shown by the examples in Section 1) that the game chromatic number is not monotone: a subgraph can have a large game chromatic number. However, it is easy to verify that the proof of Theorem 1 works for the subgraph $H$ of the Cartesian product $G \square G^{\prime}$ : if an edge $e=x y$ is deleted, then Alice just pretends the edge is still there. This may cause a problem if Alice's strategy relies on the assumption that Bob will never color $x$ and $y$ the same color. However, in the strategy described above, Alice does not need that assumption, and so this will not cause any problem. If a vertex $x$ is deleted, then Alice can still use the same strategy. In case Alice needs to color $x$ (which does not exist in reality), Alice simply colors any uncolored vertex with a correct color.

Theorem 2. Let $G$ and $G^{\prime}$ be two graphs. Assume $G^{\prime}$ has $n^{\prime}$ vertices. Let $G^{*}$ be obtained from $G$ by joining to each vertex $x$ of $G$ a set $W_{x}$ of $n^{\prime}$ degree 1 vertices. Suppose $\operatorname{Rcol}\left(G^{*}\right)=m$ and $\chi_{w a}\left(G^{\prime}\right)=k$. Then for any subgraph $H$ of $G \square G^{\prime}$ (induced or not induced), $\chi_{\mathrm{g}}^{B}(H) \leq k(k+m-1)$.

Theorem 2 can be applied to obtain upper bounds for $\chi_{\mathrm{g}}(H)$ for subgraphs $H$ of the Cartesian product of various classes of graphs.

Corollary 2. Suppose $\mathcal{H}_{1}$ and $\mathcal{H}_{2}$ are two classes of graphs. Let

$$
\mathcal{H}_{1} \square \mathcal{H}_{2}=\left\{H: H \subseteq G \square G^{\prime}, G \in \mathcal{H}_{1}, G^{\prime} \in \mathcal{H}_{2}\right\}
$$

Then the following hold:

- $\chi_{\mathrm{g}}(\mathcal{F} \square \mathcal{F}) \leq 10$.

- $\chi_{\mathrm{g}}(\mathcal{F} \square \mathcal{Q}) \leq 16$.

- $\chi_{\mathrm{g}}(\mathcal{F} \square \mathcal{P}) \leq 36$.

- $\chi_{\mathrm{g}}\left(\mathcal{F} \square \mathcal{P} K_{k}\right) \leq 6 k+6$.

- $\chi_{\mathrm{g}}(\mathcal{Q} \square \mathcal{P}) \leq 55$.

- $\chi_{\mathrm{g}}(\mathcal{P} \square \mathcal{P}) \leq 105$.

The list can be continued. They are just consequences of the combination of Theorem 2 with known upper bounds on the acyclic chromatic number and game coloring number of classes of graphs. For the results listed above, we use the bounds, that we cited before, on the game coloring numbers of forest, outerplanar graphs, partial $k$-trees, planar graphs. Note that if $G$ is a forest (or an outerplanar graph, or 
a planar graph, or a partial $k$-tree), then so is $G^{*}$. We also use the bounds on the acyclic chromatic number of graphs: planar graphs have acyclic chromatic number at most 5, outerplanar graphs have acyclic chromatic number at most 3, partial $k$-trees have acyclic chromatic number at most $k+1$, etc.

Observe that the product $G \square G^{\prime}$ and $G^{\prime} \square G$ are isomorphic. When we use Theorem 1 to derive an upper bound for $\chi_{\mathrm{g}}^{B}\left(G \square G^{\prime}\right)$, we may try both order and choose the better bound. For example, if $G$ is outerplanar and $G^{\prime}$ is a forest, then $\chi_{\mathrm{g}}^{B}\left(G \square G^{\prime}\right) \leq \min \{2 \times(7+2-1), 3 \times(4+3-1)\}$.

Observe also that the classes of graphs in Corollary 2 satisfy the assumption of Lemma 1. So we can use $\chi_{\mathrm{g}}$ in place of $\chi_{\mathrm{g}}^{B}$.

It is proved in [16] that the Cartesian product of $d$ trees has acyclic chromatic number at most $d+1$. It follows from a result in [25] that the Cartesian product of $d$ planar graphs has acyclic chromatic number at most $50(d-1)-9$. The product of two planar graphs has acyclic chromatic number at most 25 . Thus we have the following corollary:

Corollary 3. For a class $\mathcal{H}$ of graphs and for an integer $d \geq 2$, let $\square_{d} \mathcal{H}=$ $\left\{H_{1} \square H_{2} \square \cdots \square H_{d}: H_{i} \in \mathcal{H}\right\}$. Then

- $\chi_{\mathrm{g}}\left(\square_{d} \mathcal{F}\right) \leq d(d+3)$.

- $\chi_{\mathrm{g}}\left(\square_{3} \mathcal{P}\right) \leq 1025$ and for $d \geq 4, \chi_{\mathrm{g}}\left(\square_{d} \mathcal{P}\right) \leq(50 d-109)(50 d-93)$.

\section{SOME REMARKS AND QUESTIONS}

The game chromatic number of graphs has been studied extensively in the literature. However, there are very few known strategies for either Alice or Bob to play the game. The most useful strategy for Alice for proving upper bounds for the game chromatic number is the so called activation strategy. The method of using acyclic coloring to design a strategy for Alice to play the coloring game was invented in [7], where it is proved that for any graph $G, \chi_{\mathrm{g}}^{A}(G) \leq \chi_{a}(G)\left(\chi_{a}(G)+1\right)$. The strategy used in the proof of Theorem 1 blends the activation strategy and the acyclic coloring method together.

This blended strategy does have some advantage compared to the case that only one of the acyclic coloring method or the activation strategy is used. If only the acyclic coloring method is used, the upper bound obtained is not as sharp as the one given in Theorem 1. The activation strategy alone simply does not work for the Cartesian product of graphs with large maximum degree, because an upper bound derived by using the activation strategy should be an upper bound for the game coloring number, but the game coloring number of $K_{1, n} \square K_{1, n}$ is not bounded, as shown in [1]. Indeed, a result of Kierstead and Trotter [20] implies that $\operatorname{col}_{\mathrm{g}}^{B}\left(K_{1, n} \square K_{1, n}\right)$ is of the order of $\ln n$. For a graph $G$, denote by $s d_{1}(G)$ the graph obtained from $G$ by replacing each edge with a path of length 2 . Kierstead and Trotter proved that $s d_{1}\left(K_{n, n}\right)$ has game coloring number of the order of $\ln n$. As $K_{1, n} \square K_{1, n} \backslash\left\{\left(x_{0}, y_{0}\right)\right\}$ is isomorphic to $s_{1}\left(K_{n, n}\right)$ (where $x_{0}$ and $y_{0}$ are the center Journal of Graph Theory DOI 10.1002/jgt 
of the two copies of $K_{1, n}$, respectively), $\operatorname{col}_{\mathrm{g}}^{B}\left(K_{1, n} \square K_{1, n}\right)$ is also of the order of $\ln n$.

Theorem 2 implies that $\chi_{\mathrm{g}}^{B}\left(K_{1, n} \square K_{1, n^{\prime}}\right) \leq 10$. However, a slight modification of the arguments in [20] proves the following two results:

Theorem 3. For any positive integers $n, n^{\prime}, \chi_{\mathrm{g}}^{B}\left(K_{1, n} \square K_{1, n^{\prime}}\right) \leq 5$.

Theorem 4. For any positive integers $n, n^{\prime}$, for any subgraph $H$ of $s d_{1}\left(K_{n, n^{\prime}}\right)$, we have $\chi_{\mathrm{g}}^{B}(H), \chi_{\mathrm{g}}^{A}(H) \leq 4$.

The upper bound for the game chromatic number of $K_{1, n} \square K_{1, n^{\prime}}$ and for $s d_{1}\left(K_{n, n^{\prime}}\right)$ are sharp, provided that $n, n^{\prime}$ are large enough.

Theorem 5. If $n, n^{\prime}$ are large enough, then $\chi_{\mathrm{g}}^{B}\left(K_{1, n} \square K_{1, n^{\prime}}\right)=5$ and $\chi_{\mathrm{g}}^{B}\left(s d_{1}\left(K_{n, n^{\prime}}\right)\right)=4$.

Proof. We only prove it for $K_{1, n} \square K_{1, n^{\prime}}$. It suffices to show that with four colors, Bob has a winning strategy in the coloring game on $K_{1, n} \square K_{1, n^{\prime}}$. Assume the color set is $\{0,1,2,3\}$, and the vertices are named as in the proof of Theorem 3 . Bob colors $\left(x_{0}, y_{0}\right)$ with color 0 in his first move. In his next three moves, he colors vertices $\left(x_{1}, y_{1}\right),\left(x_{2}, y_{2}\right),\left(x_{3}, y_{3}\right)$ with colors $1,2,3$, respectively. Before Bob colors $\left(x_{i}, y_{i}\right)$, we assume that both $\left(x_{0}, y_{i}\right)$ and $\left(x_{i}, y_{0}\right)$ are uncolored. This assumption is no problem, because $n, n^{\prime}$ are large enough and we can permute the indices of the $x$ 's and the indices of the $y$ 's. Thus when Bob colors vertex $\left(x_{i}, y_{i}\right)$, both vertices $\left(x_{0}, y_{i}\right)$ and $\left(x_{i}, y_{0}\right)$ are "threatened." Alice can protect only one of these two vertices in one move. Thus after all the three vertices $\left(x_{1}, y_{1}\right),\left(x_{2}, y_{2}\right)$, $\left(x_{3}, y_{3}\right)$ are colored, and after Alice's response to the last attack, at least three of the six vertices $\left\{\left(x_{0}, y_{i}\right),\left(x_{i}, y_{0}\right): i=1,2,3\right\}$ are uncolored. Then two of the three uncolored vertices (among the six) belong to $X$ or $Y$. Thus without loss of generality, we assume that we arrive at the state that $\left(x_{1}, y_{1}\right),\left(x_{2}, y_{2}\right),\left(x_{3}, y_{3}\right)$ are colored by $1,2,3$, respectively, and $\left(x_{0}, y_{1}\right),\left(x_{0}, y_{2}\right)$ are uncolored, and it is Bob's turn.

By permuting the indices if necessary, we further assume that $\left(x_{4}, y_{j}\right)$ are uncolored for $j=0,1, \ldots, 6$. Bob colors $\left(x_{4}, y_{1}\right)$ with color 2 . This forces Alice to color $\left(x_{0}, y_{1}\right)$, because if not, then Bob would color a vertex, say $\left(x_{5}, y_{1}\right)$ with color 3 , and then $\left(x_{0}, y_{1}\right)$ would have no legal color.

Next Bob colors $\left(x_{4}, y_{2}\right)$ with color 1 . Now both vertices $\left(x_{0}, y_{2}\right)$ and $\left(x_{4}, y_{0}\right)$ need immediate protection, and Alice can protect only one of them. So Bob wins the game.

In the proof of Theorem 5, we assume that $n, n^{\prime}$ are large enough. By a more careful analysis, it can be shown that if $n, n^{\prime} \geq 5$, we already have $\chi_{\mathrm{g}}^{B}\left(K_{1, n} \square K_{1, n^{\prime}}\right)=5$ and $\chi_{\mathrm{g}}^{B}\left(s d_{1}\left(K_{n, n^{\prime}}\right)\right)=4$. If $n, n^{\prime} \geq 6$, then we have $\chi_{\mathrm{g}}^{A}\left(K_{1, n} \square K_{1, n^{\prime}}\right)=5$ and $\chi_{\mathrm{g}}^{A}\left(s d_{1}\left(K_{n, n^{\prime}}\right)\right)=4$.

A class $\mathcal{H}$ of graphs is hereditary if $G \in \mathcal{H}$ implies that for every subgraph $G^{\prime}$ of $G, G^{\prime} \in \mathcal{H}$. Since $\operatorname{col}_{\mathrm{g}}\left(s d_{1}\left(K_{n, n}\right)\right)$ is of the order of $\ln n$, we have a hereditary class of graphs (i.e., the subgraphs of the graphs $\left.s d_{1}\left(K_{n, n}\right)(n=1,2, \ldots)\right)$ whose game chromatic number is 4 and yet whose game coloring number is unbounded. On the 
other hand, it is not difficult to verify that any hereditary class of graphs with game chromatic number 2 has game coloring number at most 3 .

Question 1. Is there a hereditary class $\mathcal{H}$ of graphs whose game chromatic number is 3 and whose game coloring number is unbounded?

It is shown in [1] that $\chi_{\mathrm{g}}^{A}\left(K_{n, n} \square K_{m, m}\right)$ goes to infinity if $n, m$ goes to infinity. This shows that $\chi_{\mathrm{g}}^{A}\left(G \square G^{\prime}\right)$ is not bounded from above by any function of $\chi_{\mathrm{g}}^{A}(G)$, $\chi_{\mathrm{g}}^{A}\left(G^{\prime}\right)$. For a graph $G$, let

$$
\chi_{\mathrm{hg}}^{B}(G)=\max \left\{\chi_{\mathrm{g}}^{B}(H): H \text { is a subgraph of } G\right\} .
$$

The following question is open.

Question 2. Suppose $\chi_{\mathrm{hg}}^{B}(G)$ and $\chi_{\mathrm{hg}}^{B}\left(G^{\prime}\right)$ are both bounded by a constant. Is it true that $\chi_{\mathrm{hg}}^{B}\left(G \square G^{\prime}\right)$ bounded by a constant? More generally, suppose $\mathcal{H}_{1}$ and $\mathcal{H}_{2}$ are two hereditary classes of graphs. If both $\chi_{\mathrm{g}}^{B}\left(\mathcal{H}_{1}\right)$ and $\chi_{\mathrm{g}}^{B}\left(\mathcal{H}_{2}\right)$ are bounded by a constant, is $\chi_{\mathrm{g}}^{B}\left(\mathcal{H}_{1} \square \mathcal{H}_{2}\right)$ bounded by a constant?

In Theorem 1, the B-game chromatic number $\chi_{\mathrm{g}}^{B}\left(G \square G^{\prime}\right)$ is bounded in terms of $\chi_{a}\left(G^{\prime}\right)$ and $\operatorname{col}_{\mathrm{g}}^{B}\left(G^{*}\right)$, where $G^{*}$ is obtained from $G$ by adding some degree 1 vertices. Adding degree 1 vertices may increase the game coloring number of a graph by 1 . For example, $\operatorname{col}_{\mathrm{g}}^{B}\left(K_{n}\right)=\operatorname{col}_{\mathrm{g}}^{A}\left(K_{n}\right)=n$, but by joining one degree 1 vertex to each vertex of $K_{n}$, the resulting graph has game coloring number $n+1$.

Question 3. Suppose $G^{*}$ is obtained from $G$ by joining to each vertex a number of degree 1 vertices. Is it true that $\operatorname{col}_{\mathrm{g}}^{B}\left(G^{*}\right)$ and $\operatorname{col}_{\mathrm{g}}^{A}\left(G^{*}\right)$ are at most $\operatorname{col}_{\mathrm{g}}^{B}(G)+1$ and $\operatorname{col}_{\mathrm{g}}^{A}(G)+1$, respectively?

It seems difficult to prove that $\operatorname{col}_{\mathrm{g}}^{B}\left(G^{*}\right)$ is bounded from above by any function of $\operatorname{col}_{\mathrm{g}}^{B}(G)$. This leaves the following open question.

Question 4. Prove that $\chi_{\mathrm{g}}^{B}\left(G \square G^{\prime}\right)$ is bounded by a function of $\operatorname{col}_{\mathrm{g}}^{B}(G)$ and $\chi_{a}(G)$.

We have observed that the game coloring number of $\mathcal{F} \square \mathcal{F}$ is unbounded, although $\operatorname{col}_{\mathrm{g}}(\mathcal{F})=4$.

Question 5. Suppose both $\operatorname{col}_{\mathrm{g}}^{B}\left(\mathcal{H}_{1}\right)$ and $\operatorname{col}_{\mathrm{g}}^{B}\left(\mathcal{H}_{2}\right)$ are bounded by a constant. Is it true that $\chi_{\mathrm{g}}^{B}\left(\mathcal{H}_{1} \square \mathcal{H}_{2}\right)$ bounded by a constant?

It is obvious that if Question 2 has a positive answer, then Question 5 has a positive answer.

It is easy to see that for any graphs $G$ and $G^{\prime}, \chi_{a}\left(G \square G^{\prime}\right) \leq \chi_{a}(G) \chi_{a}\left(G^{\prime}\right)$. Thus if $\chi_{a}(G)=k$ and $\chi_{a}\left(G^{\prime}\right)=k^{\prime}$, then for any subgraph $H$ of $G \square G^{\prime}, \chi_{a}(H) \leq k k^{\prime}$, implying that $\chi_{\mathrm{g}}^{B}(H) \leq k k^{\prime}\left(k k^{\prime}+1\right)$. 
Question 6. Is it true the for any graph $G, \chi_{a}(G)$ is bounded from above by a function of $\operatorname{col}_{\mathrm{g}}^{B}(G)$ ?

If Question 6 has a positive answer, then both Questions 4 and 5 have positive answers. The following result is proved in [10].

Theorem 6 (Dvořák [10]). Let $\mathcal{G}$ be a hereditary class of graphs such that the chromatic number of every graph in $\mathcal{G}$ is bounded by a constant. Then the acyclic chromatic number of graphs in $\mathcal{G}$ is bounded by a constant if and only if for any graph $G$, $\operatorname{sd}_{1}(G) \in \mathcal{G}$ implies that $\chi(G) \leq c$.

The following conjecture is equivalent to a conjecture proposed in [10], and implies a positive answer to Question 6.

Conjecture 1 Dvořák ([10]). Suppose $\mathcal{G}$ is hereditary class of graphs and $\operatorname{col}_{\mathrm{g}}^{A}(H)$ is bounded by a constant for $H \in \mathcal{G}$. Then there is a constant c for any graph $G$, $s d_{1}(G) \in \mathcal{G}$ implies that $G$ has a vertex of degree at most $c$.

\section{ACKNOWLEDGMENT}

This work was supported in part by the National Science Council under grant NSC95-2115-M-110-013-MY3.

\section{REFERENCES}

[1] T. Bartnicki, B. Brešar, J. Grytczuk, M. Kovše, Z. Miechowicz, and I. Peterin, Game chromatic number of Cartesian product graphs, Electron J Combin 15(1) (2008), R72.

[2] T. Bartnicki, J. Grytczuk, H. A. Kierstead, and X. Zhu, The map colouring game, Am Math Monthly, 114 (2007), 793-803.

[3] H. L. Bodlaender, On the complexity of some coloring games, Int J Found Comput Sci 2(2) (1991), 133-147.

[4] O. V. Borodin, On acyclic colorings of planar graphs, Discrete Math 25(3) (1979), 211-236.

[5] M. Borowiecki, E. Sidorowicz, and Zs. Tuza, Game list colouring of graphs, Electron J Combin 14(1): Research Paper 26, 11 pp. (electronic), 2007.

[6] M. DeVos, G. Ding, B. Oporowski, D. P. Sanders, B. Reed, P. Seymour, and D. Vertigan, Excluding any graph as a minor allows a low tree-width 2-coloring, J Combin Theory Ser B 91(1) (2004), 25-41.

[7] T. Dinski and X. Zhu, A bound for the game chromatic number of graphs, Discrete Math 196(1-3) (1999), 109-115. 
[8] C. Dunn and H. A. Kierstead, A simple competitive graph coloring algorithm. II, J Combin Theory Ser B 90(1) (2004), 93-106. Dedicated to Adrian Bondy and U. S. R. Murty.

[9] C. Dunn and H. A. Kierstead, A simple competitive graph coloring algorithm. III, J Combin Theory Ser B 92(1) (2004), 137-150.

[10] Z. Dvořák, Asymptotical structure of combinatorial objects, Ph.D. Thesis, Charles University, 2007.

[11] U. Faigle, U. Kern, H. A. Kierstead, and W. T. Trotter, On the game chromatic number of some classes of graphs, Ars Combin 35 (1993), $143-150$.

[12] M. Gardner, Mathematical Games, Scientific American, 1981.

[13] B. Grünbaum, Acyclic colorings of planar graphs, Israel J Math 14 (1973), 390-408.

[14] D. J. Guan and X. Zhu, Game chromatic number of outerplanar graphs, J Graph Theory 30(1) (1999), 67-70.

[15] W. He, X. Hou, K.-W. Lih, J. Shao, W. Wang, and X. Zhu, Edge-partitions of planar graphs and their game coloring numbers, J Graph Theory 41(4) (2002), 307-317.

[16] R. E. Jamison, G. L. Matthews, and J. Villalpando, Acyclic colorings of products of trees, Inform Process Lett 99(1) (2006), 7-12.

[17] H. A. Kierstead, A simple competitive graph coloring algorithm, J Combin Theory Ser B 78(1) (2000), 57-68.

[18] H. A. Kierstead, Weak acyclic coloring and asymmetric coloring games, Discrete Math 306(7) (2006), 673-677.

[19] H. A. Kierstead and W. T. Trotter, Planar graph coloring with an uncooperative partner, J Graph Theory 18(6) (1994), 569-584.

[20] H. A. Kierstead and W. T. Trotter, Competitive colorings of oriented graphs, Electron J Combin 8(2): Research Paper 12, 15 pp. (electronic), 2001. In honor of Aviezri Fraenkel on the occasion of his 70th birthday.

[21] H. A. Kierstead and Zs. Tuza, Marking games and the oriented game chromatic number of partial $k$-trees, Graphs Combin 19(1) (2003), 121-129.

[22] H. A. Kierstead and D. Yang, Orderings on graphs and game coloring number, Order 20(3) (2003), 255-264.

[23] H. A. Kierstead and D. Yang, Very asymmetric marking games, Order 22(2) (2005), 93-107.

[24] J. Nešetřil and E. Sopena, On the oriented game chromatic number, Electron J Combin 8(2): Research Paper 14, 13 pp. (electronic), 2001. In honor of Aviezri Fraenkel on the occasion of his 70th birthday.

[25] A. Pór and D. Wood, Colorings of the Cartesian product of graphs and multiplicative sidon sets, Manuscript, 2005. 
[26] J. Wu and X. Zhu, Lower bounds for the game colouring number of partial $k$-trees and planar graphs, Discrete Math (2007), doi:10.10106/ j.disc.2007.05.023.

[27] X. Zhu, The game coloring number of planar graphs, J Combin Theory Ser B 75(2) (1999), 245-258.

[28] X. Zhu, The game coloring number of pseudo partial $k$-trees, Discrete Math 215(1-3) (2000), 245-262.

[29] X. Zhu, Refined activation strategy for the marking game, J Combin Theory Ser B (2007), doi: 10.1016/j.jctb.2007.04.004. 